\title{
Perceptions of the IQOS Heated Tobacco Product on Twitter in the United States
}

\author{
Canruo Zou ${ }^{1}$, Xueting Wang $^{2}$, Zidian Xie ${ }^{3 *}$ and Dongmei $\mathrm{Li}^{3 *}$ \\ ${ }^{1}$ Department of Computer Science, University of Rochester, Rochester, NY, United States, ${ }^{2}$ Goergen Institute for Data Science, \\ University of Rochester, Rochester, NY, United States, ${ }^{3}$ Department of Clinical and Translational Research, University of \\ Rochester Medical Center, Rochester, NY, United States
}

OPEN ACCESS

Edited by:

Seow Ting Lee,

University of Colorado Boulder,

United States

Reviewed by:

Douglas Ashwell,

Massey University Business School,

New Zealand

Brittany N. Lash,

University of Dayton, United States

${ }^{*}$ Correspondence:

Zidian Xie

Zidian_Xie@urmc.rochester.edu

Dongmei Li

Dongmei_Li@urmc.rochester.edu

Specialty section:

This article was submitted to

Health Communication,

a section of the journal

Frontiers in Communication

Received: 21 June 2021

Accepted: 26 July 2021

Published: 20 August 2021

Citation:

Zou C, Wang X, Xie Z and Li D (2021)

Perceptions of the IQOS Heated

Tobacco Product on Twitter in

the United States.

Front. Commun. 6:728604.

doi: 10.3389/fcomm.2021.728604
After the approval of the sales of IQOS in the United States market, discussions about IQOS have become active on social media. Twitter is a popular social media platform to understand public opinions toward IQOS. This study aims to explore public perceptions toward IQOS on Twitter in the United States. IQOS-related tweets from the United States between November 19, 2019, and August 24, 2020, were collected using a Twitter streaming application programming interface (API). Sentiment analysis was performed to determine whether the public perceptions toward IQOS were positive, neutral, or negative. In addition, topics discussed in these tweets were manually coded. From November 2019 to August 2020, the number of tweets discussing IQOS was relatively constant except for a peak starting from July 7, 2020, which lasted for 4 days. Among IQOS tweets with positive sentiments, the most popular topic is "IQOS is safer than cigarettes," followed by "IQOS helps quit smoking." Among tweets with negative sentiments, the most popular topic is "illegal marketing/selling to youth," followed by "health risks/fire hazards." "FDA approval/ regulation" is the most popular topic for tweets with neutral sentiments. After the announcement of the United States Food and Drug Administration (FDA) enforcement policy on unauthorized flavored e-cigarette products on January 2, 2020, the proportion of tweets with positive attitudes toward IQOS significantly increased, while the proportion of negative tweets significantly decreased. Our study showed that the public perception of IQOS in the United States became more positive after the FDA enforcement policy on flavored e-cigarettes. While many Twitter users thought IQOS is safer than cigarettes and helps quit smoking, some Twitter users complained about the illegal marketing and health risks of IQOS. These findings provide useful information on future tobacco regulations.

Keywords: iQOS, twitter, social media, perception, tobacco and tobacco product

\section{INTRODUCTION}

IQOS is a new heated tobacco product manufactured and promoted by the tobacco giant Philip Morris International (PMI). Compared to e-cigarettes that deliver nicotine to the users by a heated liquid, IQOS heats real tobacco leaves that contain toxins and harmful substances (Underferth, 2019). After years of regulatory debate, on April 30, 2019, the U.S. Food and Drug Administration (FDA) approved the sale of IQOS in the United States market, which required PMI to follow stringent marketing restrictions to prevent youth access and exposure. Meanwhile, the discussion of 
IQOS's health effects, such as whether IQOS reduces exposure to harmful components, was frequently brought to scholarly reviews and public attention. A research report from Tobacco Control showed that adult consumers perceived the reduced-exposure claims as reduced-risk claims, which were not scientifically true (Lucy, 2018). On July 07, 2020, the FDA authorized the marketing of IQOS as a "reduced-exposure" tobacco product, with scientific evidence showing a significant reduction of harmful or potentially harmful chemical exposures if a conventional cigarette smoker completely switched to the IQOS system (RELEASE, 2020). The FDA is also closely monitoring the dynamics of the IQOS market to ensure the sales of IQOS in the U.S. are appropriate and the marketing restrictions are being followed to prevent youth access and exposure (Office of the, 2020; RELEASE, 2020).

Social media platforms such as Twitter and Instagram are popular for the public to share their opinions. A study by Hejlova (2019) concluded that advertisement by the tobacco industry has misguided the rapid evolution of social media, especially Instagram. Twitter is an online microblogging and networking platform where users can post tweets to deliver messages and express thoughts. Twitter data had been used to examine the public perceptions of different e-liquid flavors through sentiment analysis (Lu, 2020). Another study on disposable electronic cigarettes showed that e-cigarette companies were promoting flavored disposable e-cigarettes to young people on Twitter by taking advantage of loopholes (Li Sun, 2020). A recent study categorizing IQOS-related tweets (from January 1, 2020, to June $30,2020)$ found that Twitter discussions focused on marketing, personal testimonials, and also health claims (Barker et al., 2021). With the growth of attention toward IQOS, including marketing and sales, FDA regulation policy, and health effects, the IQOSrelated topics and their public perception may vary over time. In this study, we aimed to explore the longitudinal trend of IQOS discussion and sentiments toward IQOS on Twitter in the United States. Our study provides valuable information about public attitudes toward the launch and regulation of IQOS in the United States, which could guide future research on exploring related fields and provide important information for FDA regulatory science.

\section{MATERIALS AND METHODS}

\section{Twitter Data Collection and Preprocessing}

The Twitter dataset on IQOS used for this study was crawled from November 19, 2019, to August 24, 2020, by using the Twitter streaming API. ${ }^{1}$ The keywords that were used to crawl the IQOSrelated tweets were "iqos," "heatnotburn," and "heatedtobacco." "heatnotburn" and "heatedtobacco" usually refer to IQOS on social media. The total number of IQOS-related tweets was 31,746. Keywords "dealer," "deal," “supply," “store," "promo," "promotion," "customer," "discount," "sale," “free shipping," "\$," "\%," "dollar," "offer," "percent off," "save," "price," and "wholesale" were used to remove the commercial tweets (Gao

\footnotetext{
${ }^{1}$ Twitter API | Products.
}

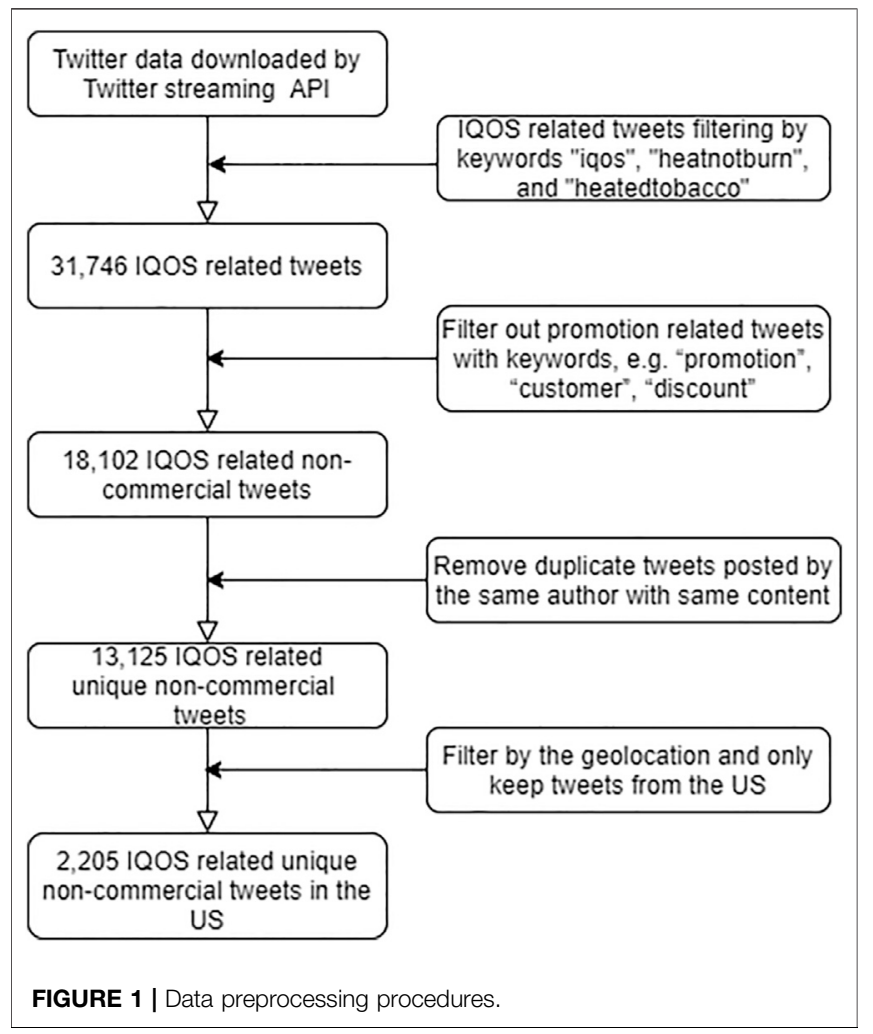

et al., 2021). Among them, there were 13,644 commercial tweets and 18,102 noncommercial tweets. Duplicated tweets that were published by the same user and with the same content were removed. The number of unique IQOS-related tweets was 13,125. The names of the country, state, and cities with the top 50 population, such as "New York, New York" for New York City and "California" for California state, were used to filter tweets with valid geolocation in the United States (Bureau, 2010). The total number of unique noncommercial IQOS-related tweets from the United States between November 19, 2019, and August 24, 2020, was 2,205 (Figure 1).

\section{Temporal Analysis}

A temporal analysis was performed on the tweets to learn how the tweets changed longitudinally during the study period. The number of tweets on IQOS each week was calculated to investigate the temporal trend of IQOS discussions on Twitter from the United States.

\section{Content Analysis}

Sentiment analysis was conducted to learn the attitude toward IQOS in the tweets by manually coding the tweets into different sentiment categories. Two team members individually handcoded the sentiments of the tweets as either positive, neutral, or negative attitude toward IQOS. For example, "IQOS is fantastic" will be classified as positive, "IQOS store is open" will be classified as neutral, and "IQOS causes cancer" will be classified as negative. The agreement rate between the two individual coders was about $78.9 \%$ with Cohen's kappa 
statistic of 0.72 , indicating a substantial agreement between the two coders (McHugh, 2012). Any disagreement was resolved by the discussion within the study team with four members. The FDA issued a policy prioritizing enforcement against unauthorized cartridge-based flavored e-cigarette products except for tobacco and menthol flavors (the FDA flavor ban) on January 2, 2020 (Commissioner, 2020). A two-proportion Z-test was conducted to compare the proportion of tweets with different sentiment categories between before (November 19, 2019-January 1, 2020) and after (January 2, 2020-August 24, 2020) the FDA flavor ban.

Topic analysis was manually conducted by two individual coders to group IQOS-related tweets into different topic categories. Two hundred tweets were randomly selected to determine the topics and reliability by two independent coders, which were used to classify the remaining tweets. The main topics of these tweets were summarized in each sentiment group. Topics were explained as the following. Among tweets with positive sentiments, "IQOS is better than cigarette" meant IQOS is better or safer than a traditional cigarette. "IQOS helps quit smoking" meant IQOS helps quit smoking traditional cigarettes. "Support FDA approval" meant supporting FDA's regulations about IQOS. "IQOS reduces exposure to harmful chemicals" meant IQOS reduces exposure to harmful chemicals compared to traditional cigarettes or e-cigarettes. "IQOS is better than e-cigarette" meant IQOS is better or safer than an e-cigarette. "Positive others" meant tweets that could not be categorized in any identified topics. Among tweets with neutral sentiments, "FDA approval/regulation" meant FDA's regulations about IQOS or other e-cigarettes. "Financial market/PMI stock" meant financial securities and derivatives of IQOS such as Philip Morris International's stock. "Sales and taxes" meant the sale of IQOS and taxation on IQOS. "Debate between FDA and other parties" meant the FDA and other parties such as the World Health Organization had different opinions on IQOS. "Neutral others" meant tweets that could not be categorized in any identified topics. Among tweets with negative sentiments, "illegal marketing/selling to youth" meant marketing or selling IQOS to youth. "IQOS health risks/fire hazards" meant health risks or fire hazards of using IQOS. "Against FDA approval” meant against FDA's regulations about IQOS or other e-cigarettes. "E-cigarette is better than IQOS" meant e-cigarette is better or safer than IQOS. "Complain about the IQOS research funded by cigarette company" meant complaining about the research on IQOS was funded by cigarette companies. "Misrepresented information in IQOS marketing" meant incorrect information used in IQOS marketing that made people want to buy it. "IQOS kills vaping industry" meant the regulation on e-cigarettes that made IQOS own the vaping market. "IQOS patent suits" meant British American Tobacco filed patent suits against IQOS. "Marketing IQOS as affluent lifestyle" meant using IQOS was marketed as an affluent lifestyle. "IQOS costs more than e-cigarette" meant buying IQOS costs more than buying an e-cigarette or a traditional cigarette. "Negative others" meant tweets that could not be categorized in any identified topics. Even though the "others" categories have many tweets, due to too many topics in them, we combined them into the others categories. If a tweet contained multiple topics, it would be classified into the primary topic because we only focused on the major theme of each tweet. Cohen's kappa statistic between two coders was around 0.72, interpreted as good (Altman, 1991). Coders discussed the difference between their coding to resolve any disagreements. The proportion of the number of tweets in each topic before and after the FDA flavor ban during the research period was calculated to examine the change in topic prevalence before and after the FDA flavor ban.

\section{RESULTS}

\section{Prevalence of IQOS Discussion on Twitter in the United States Over Time}

To examine the prevalence of IQOS discussion on Twitter, we collected and analyzed IQOS-related tweets in the United States from November 19, 2019, to August 24, 2020. As shown in Figure 2, we observed two small peaks and one large peak on the weekly number of IQOS-related tweets. Two small peaks are at the beginning of December 2019 and mid-February 2020, while the big peak is around July 07,2020 . The weekly number of IQOSrelated tweets remained relatively low at other times.

\section{Public Perceptions of IQOS on Twitter}

With the active discussion of IQOS on Twitter, it is important to examine public perceptions of IQOS on Twitter in the United States. Figure 3 shows the result of the sentiment analysis toward IQOS on Twitter in the United States. There were 383 positive tweets, 744 neutral tweets, and 1,078 negative tweets during the study period. The weekly number of positive tweets was large in the week of July 07, 2020. There were 132 positive tweets at the week of July 07, 2020 (34.7\% of total positive tweets), while the weekly number of IQOS-related tweets remained below 20 most of the time during the study period. The number of negative tweets was high before December 24, 2019, and from June 30, 2020, to July 14, 2020. The highest number of negative tweets (169 tweets) was observed in the week of December 10, 2019, and the second-highest number of negative tweets (131 tweets) was observed in the week of July 07,2020 . The numbers of negative tweets were relatively low (less than 80 tweets) in other weeks.

\section{Topics of IQOS Discussed on Twitter}

While there were different attitudes (negative, positive, and neutral) toward IQOS on Twitter, it is important to understand what these tweets were discussing. Therefore, we performed content analysis. As shown in Table 1, among positive tweets, "IQOS is better than cigarette" was the most popular topic (84 tweets), followed by "IQOS helps quit smoking" (57 tweets) and "support FDA approval" (51 tweets). Among negative tweets, "illegal marketing/selling to youth" was the most popular topic (185 tweets), followed by "IQOS health risks/fire hazards" (153 tweets) and "against FDA approval" (144 tweets). 


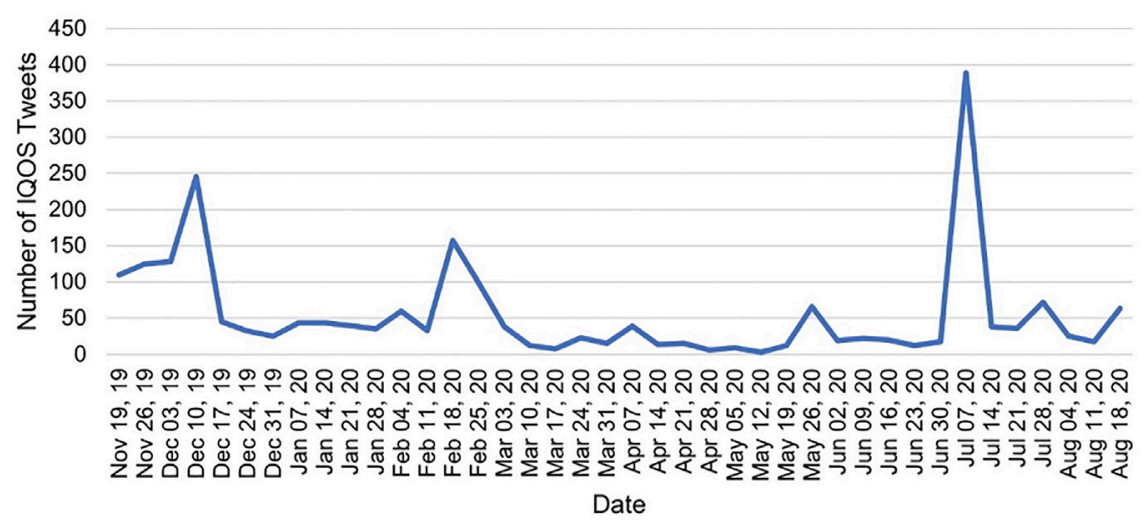

FIGURE 2 | Number of IQOS tweets in the U.S. over time.

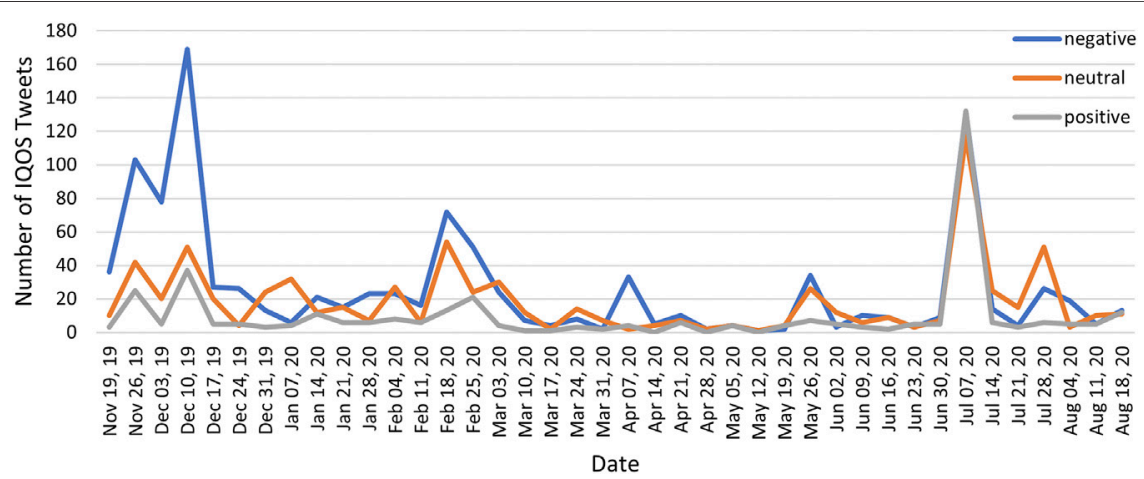

FIGURE 3 | The sentiments toward IQOS on Twitter over time.

TABLE 1 | Topics about IQOS on Twitter.

Sentiment

Positive

Negative

\section{Topic}

IQOS is better than cigarette IQOS helps quit smoking

Support FDA approval

IQOS reduces exposure to harmful chemicals

IQOS is better than e-cigarette

Positive others

FDA approval/regulation

Financial market/PMl stock

Sales and taxes

Debate between FDA and other parties

Neutral others

Illegal marketing/selling to youth

IQOS health risks/fire hazards

Against FDA approval

E-cigarette is better than IQOS

Complain about the IQOS research funded by cigarette company

Misrepresented information in IQOS marketing

IQOS kills vaping industry

IQOS patent suits

Marketing IQOS as affluent lifestyle

IQOS costs more than e-cigarette

Negative others
Number of tweets

Proportion (\%)

84
57
51
34
17
141
245
90
79
15
314
185
153
144
126
80
55
54
44
13
9
215

21.88

14.84

13.28

8.85

4.43

36.72

32.97

12.11

10.63

2.02

42.26

17.16

14.19

13.36

11.69

7.42

5.10

5.01

4.08

1.21

0.83

19.94 


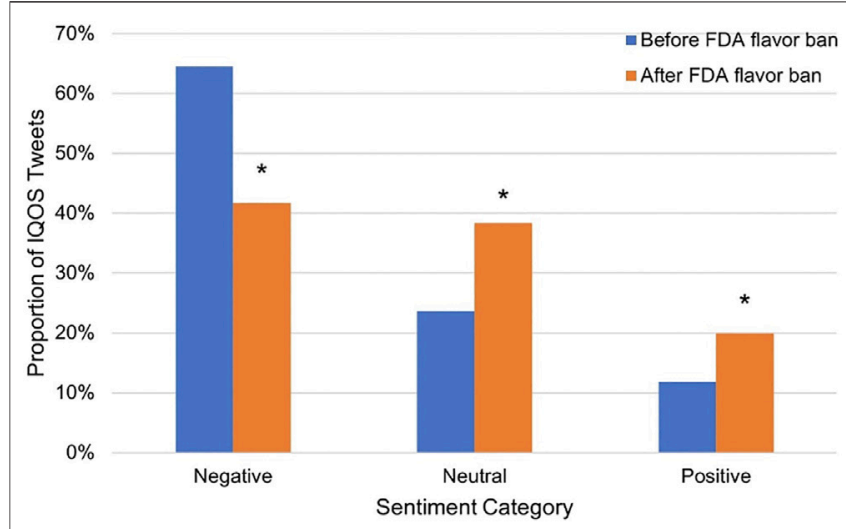

FIGURE 4 | The impact of the FDA flavor ban on the perceptions of IQOS on Twitter. ${ }^{*} p$ value $<0.0001$

Among neutral tweets, "FDA approval/regulation" was the most popular topic (245 tweets), followed by "financial market/PMI stock" (90 tweets) and "sales and taxes" (79 tweets).

\section{The Impact of the Food and Drug Administration Flavored E-Cigarette Enforcement Policy on IQOS Perception}

On January 2, 2020, the United States Food and Drug Administration (FDA) announced an enforcement policy on unauthorized cartridge-based flavored e-cigarette products except for tobacco and menthol flavors (the FDA flavor ban). To examine the possible impact of the FDA flavor ban on the public perception of IQOS, we decided to compare the sentiment of IQOS-related tweets before and after this policy. As shown in Figure 4, the proportion of tweets with negative sentiments after the FDA flavor ban was significantly lower than that before the FDA flavor ban $(p$ value $<0.0001)$. In contrast, the proportion of tweets with positive sentiments after the FDA flavor ban was significantly higher than that before the FDA flavor ban ( $p$ value $<$ $0.0001)$. In addition, the proportion of tweets with neutral sentiments after the FDA flavor ban was significantly higher than that before the FDA flavor ban ( $p$ value $<0.0001)$.

While we have shown that the proportion of tweets with different sentiments was significantly different between before and after the FDA flavor ban, it becomes important to check if the topics of these IQOS tweets have changed. Table 2 shows the proportion of each topic in each sentiment group before and after the FDA enforcement policy on flavored e-cigarettes. In the positive sentiment group, the biggest decrease in the proportion of tweets was "support FDA approval" (decreased $26.66 \%$ ), while the biggest increase was "IQOS helps quit smoking" (increased 7.41\%) besides "positive others." The biggest decrease in the proportion of tweets in the negative sentiment group was "illegal marketing/selling to youth" (decreased 10.16\%), while the biggest increase was "IQOS health risks/fire hazards" (increased 26.66\%). In addition, the biggest decrease in the proportion of tweets in the neutral sentiment group was "financial market/PMI stock" (decreased $14.81 \%$ ), while the biggest increase was "FDA approval/ regulation" (increased 26.81\%).

We have observed an active discussion on IQOS on Twitter from July 7, 2020, to July 10, 2020, which corresponds to the highest peak in Figure 2. To understand what happened during this period, we performed a content analysis on these tweets. As shown in Figure 5, excluding "positive others," the top six topics

TABLE 2 | Topics about IQOS on Twitter before and after the FDA flavor ban.

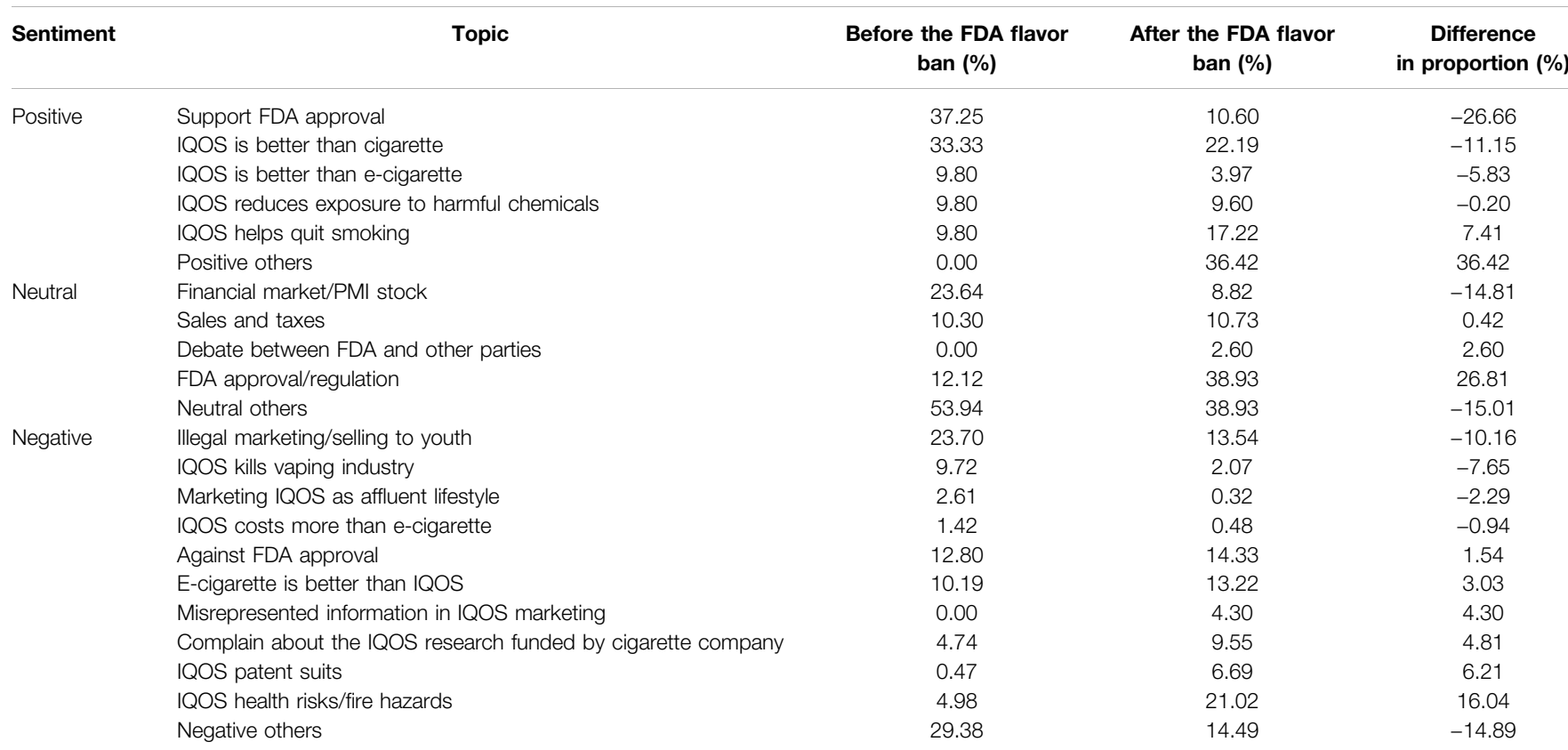




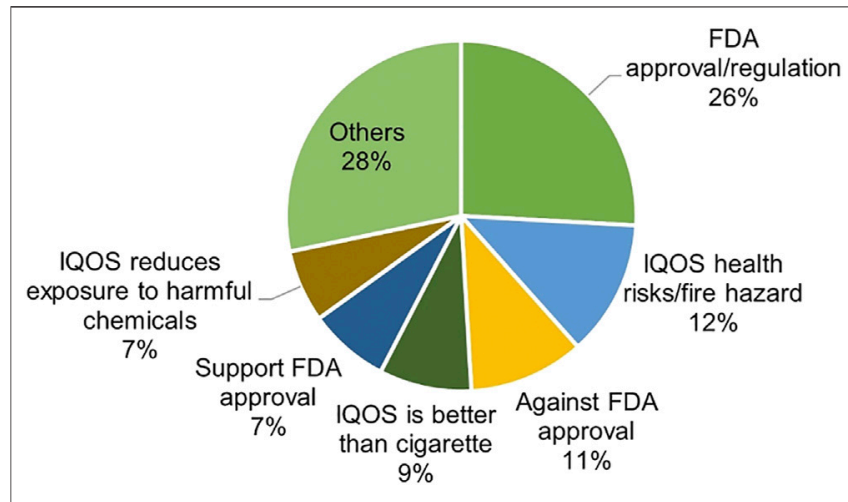

FIGURE 5 | Topics about IQOS between July 7, 2020, and July 10,2020

were "FDA approval/regulation" (26\%), "IQOS health risks/fire hazards" (12\%), "against FDA approval" (11\%), "IQOS is better than cigarette" (9\%), "support FDA approval" (7\%), and "IQOS reduces exposure to harmful chemicals" (7\%). Within the top six topics, the proportion of both positive topics and negative topics was $23 \%$.

\section{DISCUSSIONS AND CONCLUSION}

\section{Discussions}

In this study, by examining IQOS-related tweets in the United States, we showed that the prevalence of IQOS discussion on Twitter varied over time. The two small peaks and one large peak observed during our study period might be associated with events related to tobacco products. For example, the small peak in December 2019 might be related to the Tobacco 21 federal law that was announced on December 20, 2019, which raised the minimum age of tobacco use from 18 to 21 years. The other small peak in mid-February 2020 might be associated with the FDA flavor enforcement policy that was implemented on February 6, 2020, which restricted the sale of unauthorized flavored cartridge-based electronic cigarette products other than tobacco and menthol flavors. The large peak in early July 2020 corresponded to the authorization of IQOS as a "reducedexposure" product by the FDA.

While IQOS tweets with negative sentiments were dominant, we observed there was a significant decrease in the proportion of negative tweets after the FDA flavor ban. Among positive tweets, "IQOS is better than cigarette" was the most popular topic. "Illegal marketing/selling to youth" was the most popular topic within negative tweets. We observed the proportion of each IQOS-related topic has changed after the announcement of the FDA enforcement policy on flavored e-cigarettes.

Our study showed that negative tweets on IQOS significantly decreased, while positive tweets significantly increased after the FDA flavor ban. This change might be due to the FDA enforcement policy on the sale of flavored e-cigarette products to reduce youth e-cigarette use that was announced and implemented in early 2020 (Commissioner, 2020). The ban on flavored e-cigarettes might reduce the negative perception of IQOS, and more people might think that IQOS could help quit smoking (Table 2).

We observed an obvious peak in the number of IQOS tweets posted in early July 2020 . This peak occurred during the week of July 07, 2020, when the FDA authorized the marketing of IQOS as a "reduced-exposure" product. Further analysis of the tweets posted from July 07 to July 10, 2020, showed the same percentage of positive tweets and negative tweets. The topics in positive tweets in this peak period focus on "IQOS is better than cigarette," "support FDA approval," and "IQOS reduces exposure to harmful chemicals." On the contrary, the topics in negative tweets in this peak period focus on "IQOS health risks/ fire hazards" and "against FDA approval." A relatively large percentage of tweets expressed concerns about the health risks and hazards that IQOS could bring. An independent analysis of publicly available raw data from PMI's Modified Risk Tobacco Product applications showed that IQOS may reduce exposure to harmful substances; however, it did not show significant improvement in pulmonary inflammation cigarette smokers who switched to IQOS (Farzad, 2018). Although there has been some evidence showing that IQOS is safer than cigarettes (Nham Tran, 2020), there is still a significant percentage of tweets falling into this category. Future studies should investigate the potential health risks of IQOS.

Our study showed that Twitter users, in general, showed a more negative or neutral attitude toward IQOS than a positive attitude. Moreover, we showed that more tweets were mentioning "an e-cigarette is better than IQOS" than those mentioning "IQOS is better than an e-cigarette." A recent examination of heated tobacco products (HTPs), such as IQOS, related tweets from April 28, 2019, to July 1, 2019, found that tweets were more likely to be neutral or anti-HTPs than pro-HTPs regardless of the author type, and more tweets described HTPs as inferior to e-cigarettes than equal or superior to e-cigarettes (Jun 2020). Although more tweets mentioned "an e-cigarette is better than IQOS," research studies comparing health risks of IQOS with e-cigarettes are still ongoing. Maloney et al. (2020) found that IQOS reduced abstinence symptoms more effectively than JUUL (an e-cigarette brand), while Sohal et al. (2019) showed that IQOS exposure impairs human airway cell homeostasis similar to an e-cigarette. How these research findings affected Twitter users' attitude toward IQOS requires further investigation.

\section{Limitations}

There were several limitations in our study. First, we only collected data from Twitter, and only a small proportion of tweets $(\sim 67 \%)$ have valid geolocation. In addition, we did not have access to private accounts, and we only analyzed English language posts. Therefore, the findings based on the tweets we collected may not represent the whole population of IQOS users in the United States. Second, our study period was from November 18, 2019, to August 25, 2020. IQOS is a relatively new tobacco product and was permitted to sell in the United States in April 2019 (Office of the, 2020). Therefore, the public perception of IQOS on Twitter may evolve. Third, we did not examine posts related to IQOS in other social media 
platforms such as Instagram, TikTok, and YouTube, which might provide different results. Thus, our current results might not be an accurate representation of the U.S. population.

\section{CONCLUSION}

Through analyzing IQOS-related tweets in the United States from November 2019 to August 2020, we showed that the prevalence of IQOS tweets with positive sentiments increased, while the negative perception of IQOS decreased after the announcement of the FDA flavor enforcement policy on e-cigarettes. Topic analysis showed that the IQOS tweets with positive sentiments mainly focus on the topics of "IQOS is better than cigarette" and "IQOS helps quit smoking," while the IQOS tweets with negative sentiments mainly focus on the topics of "illegal marketing/selling to youth" and "IQOS health risks/fire hazards." Previous studies had shown that attitude changes could lead to potential behavior change (Price, 2015; Sheeran et al., 2016). Our study provided preliminary data that the FDA flavor ban on e-cigarettes could drive the public perception of IQOS to the positive side, which might lead to potential tobacco user's behavior changes such as the initiation of IQOS use or switching from e-cigarette to IQOS. Given the relatively short time of IQOS in the United States market, further investigation on potential health risks associated with IQOS use will be important. In addition, continued monitoring of the IQOS marketing on

\section{REFERENCES}

Altman, D. G. (1991). Practical Statistics for Medical Research. Lodon: Chapman \& Hall.

Barker, J. O., Vassey, J., Chen-Sankey, J. C., Allem, J.-P., Cruz, T. B., and Unger, J. B. (2021). Categorizing IQOS-Related Twitter Discussions. Int. J. Environ. Res. Public. Health. 18 (9), 4836. doi:10.3390/ijerph18094836

Bureau, U. S. C. City and Town Population Totals: 2010-2019. The United States Census Bureau.

Commissioner, O. o. t. (2020). FDA Finalizes Enforcement Policy on Unauthorized Flavored Cartridge-Based E-Cigarettes that Appeal to Children, Including Fruit and Mint. FDA.

Farzad, M. (2018). Assessment of Industry Data on Pulmonary and Immunosuppressive Effects of IQOS. London: Tobacco Control.

Gao, Y., Xie, Z., and Li, D. (2021). Electronic Cigarette Users' Perspective on the COVID-19 Pandemic: Observational Study Using Twitter Data. JMIR Public Health Surveill. 7 (1), e24859. doi:10.2196/24859

Hejlová, D. (2019). Analysis of Presumed IQOS Influencer Marketing on Instagram in the Czech Republic in 2018-2019. ADIKTOLOGIE J. 1, 7-15.

Jun, J. (2020). Social Response to the FDA Authorization of Heated Tobacco Products (HTPs). Tob. Regul. Sci. 6 (1), 20-29. doi:10.18001/trs.6.1.3

Li Sun, C. T. (2020). Promotion of Disposable Electronic Cigarette Flavors and Topics on Twitter. NCBI: International Journal of Environmental.

Lu, X. (2020). User Perceptions of Different Electronic Cigarette Flavors on Social Media: Observational Study. J. Med. Internet Res. 22 (6), e17280. doi:10.2196/17280

Lucy, P. (2018). Light and Mild Redux: Heated Tobacco Products' Reduced Exposure Claims Are Likely to Be Misunderstood as Reduced Risk Claims. London: Tobacco Control.

Maloney, S., (2020). Acute Effects of JUUL and IQOS in Cigarette Smokers. London: Tobacco Control.

McHugh, M. L. (2012). Interrater Reliability: the Kappa Statistic. Biochem. Med. 22 (3), 276-282. doi:10.11613/bm.2012.031 social media and assessing of its impact on user behavior change will be critical to inform FDA's regulatory science.

\section{DATA AVAILABILITY STATEMENT}

Publicly available datasets were analyzed in this study. These data can be found here: the datasets analyzed for this study are publicly available from Twitter. Further inquiries can be directed to the corresponding authors.

\section{AUTHOR CONTRIBUTIONS}

Conceptualization, ZX and DL; formal analysis, CZ and XW; resources, $\mathrm{ZX}$; data curation, $\mathrm{CZ}$ and $\mathrm{XW}$; writing-original draft preparation, $\mathrm{CZ}$ and XW; writing-review and editing, ZX and $\mathrm{DL}$; visualization, CZ; supervision, $\mathrm{ZX}$ and $\mathrm{DL}$; funding acquisition, DL.

\section{FUNDING}

This research was funded by the National Cancer Institute of the National Institutes of Health (NIH) and the U.S. Food and Drug Administration (FDA) Center for Tobacco Products under award number U54CA228110.

Nham Tran, F. D. (2020). What Are 'Heat Not Burn' Products and Are They Any Safer than Traditional Cigarettes. The Conversation. Available at.

Office of the C. (2020). FDA Permits Sale of IQOS Tobacco Heating System through Premarket Tobacco Product Application Pathway. FDA.

Price B. (2015). Understanding Attitudes and Their Effects on Nursing Practice. Nurs. Stand. 30 (15), 50-60. doi:10.7748/ns.30.15.50.s51

Release, F. N. (2020). FDA Authorizes Marketing of IQOS Tobacco Heating System with 'Reduced Exposure' Information. The Conversation.

Sheeran, P., Maki, A., Montanaro, E., Avishai-Yitshak, A., Bryan, A., Klein, W. M. P., et al. (2016). The Impact of Changing Attitudes, Norms, and Self-Efficacy on Health-Related Intentions and Behavior: A Meta-Analysis. Health Psychol. 35 (11), 1178-1188. doi:10.1037/hea0000387

Sohal, S. S., (2019). IQOS Exposure Impairs Human Airway Cell Homeostasis: Direct Comparison with Traditional Cigarette and E-Cigarette. ERJ Open Res. 5 (1). doi:10.1183/23120541.00159-2018

Underferth, D. (2019). IQOS: What You Need to Know. Anderson Cancer Center.

Conflict of Interest: The authors declare that the research was conducted in the absence of any commercial or financial relationships that could be construed as a potential conflict of interest.

Publisher's Note: All claims expressed in this article are solely those of the authors and do not necessarily represent those of their affiliated organizations or those of the publisher, the editors, and the reviewers. Any product that may be evaluated in this article, or claim that may be made by its manufacturer, is not guaranteed or endorsed by the publisher.

Copyright (C) 2021 Zou, Wang, Xie and Li. This is an open-access article distributed under the terms of the Creative Commons Attribution License (CC BY). The use, distribution or reproduction in other forums is permitted, provided the original author(s) and the copyright owner(s) are credited and that the original publication in this journal is cited, in accordance with accepted academic practice. No use, distribution or reproduction is permitted which does not comply with these terms. 